\title{
Radio resource management for uplink carrier aggregation in LTE-Advanced
}

\author{
Hua Wang ${ }^{1 *}$, Claudio Rosa ${ }^{2}$ and Klaus I Pedersen ${ }^{1,2}$
}

\begin{abstract}
This paper investigates the uplink resource allocation problem in the context of Long-Term Evolution (LTE)-Advanced systems with carrier aggregation (CA) and dual-cluster scheduling. On one hand, these Rel'10 functionalities can increase the available transmission bandwidth and scheduling flexibility in uplink. On the other hand, they will introduce additional power back-off for the power amplifier in the user equipment (UE) with non-contiguous resource allocation. Taking into account that the uplink is inherently limited by the maximum transmission power of the UE, the assignment of uplink CA and/or dual cluster transmission for LTE-Advanced UEs has to be careful. A pathloss-threshold-based component carrier (CC) and cluster configuration algorithm is proposed to determine whether an LTE-Advanced UE should be configured with multiple CCs and/or dual cluster scheduling. An extended bandwidth-expansion-based packet scheduling algorithm is proposed for dual-cluster transmission, which tightly couples the bandwidth allocation and packet scheduling together to exploit the frequency domain diversity with low complexity. Simulation results show that with proper differentiation between power-limited and non-power-limited UEs, Rel'10 CA with dual-cluster scheduling can maintain similar coverage performance as in Rel'8 case while achieving substantial gains in median and peak user throughputs. Moreover, the proposed CC assignment algorithm achieves higher user throughput as compared to blindly assigning all UEs on all available CCs.
\end{abstract}

\section{Introduction}

Carrier aggregation (CA) is one of the key features for Long-Term Evolution (LTE)-Advanced to support higher transmission bandwidth up to $100 \mathrm{MHz}$, enabling peak date rates requirements of up to 1 Gbps in downlink and $500 \mathrm{Mbps}$ in uplink to be satisfied. This is achieved by aggregating two or more individual component carriers (CCs) of the same or different bandwidth belonging to contiguous or non-contiguous frequency bands, subject to spectrum availability and the user equipment (UE)'s capability [1-5]. In addition to bandwidth extension enabled by $\mathrm{CA}$, dual-cluster transmission has also been introduced in Rel'10 to further improve the spectral efficiency in uplink. With dualcluster transmission, within a CC, a UE can be allocated to a maximum of two non-contiguous clusters, each of which includes one or more consecutive radio resource blocks [6,7]. Dual-cluster scheduling has the advantage of higher scheduling flexibility as compared to single carrier

\footnotetext{
* Correspondence: huw@es.aau.dk

'Aalborg University, Fredrik Bajers Vej 7, DK-9220 Aalborg Ø, Denmark Full list of author information is available at the end of the article
}

frequency division multiple access (SC-FDMA), while keeping the peak-to-average power ratio (PAPR) or signal cubic metric $(\mathrm{CM})$ relatively low as compared to orthogonal frequency division multiple access (OFDMA).

In the open literature, there are quite many studies on the topic of CA. The performance analysis of downlink $\mathrm{CA}$ is investigated in [8-10]. Enhancements of downlink control channel resource allocation and mobility management for CA are studied in [11-13]. Different carrier load balancing schemes and packet scheduling algorithms are analyzed in [14] by means of theoretical formulations as well as system level simulations. Enhanced frequency diversity schemes for CA are exploited in [15-17]. However, most of the existing work on CA for LTE-Advanced is mainly concentrated on the downlink. There are a few studies in the uplink. An overview of uplink multiple access transmission schemes in support of CA is provided in [18]. A dynamic uplink CA scheme is proposed in [19] to improve the energy efficiency of uplink communications. An edge-prioritized channel and traffic aware CC assignment resource and allocation algorithm is proposed in [20]. The performance 
of uplink CA in LTE-Advanced systems with different CC allocation schemes is investigated in [21-24].

As different CCs may operate at different frequencies and bandwidths, questions arise as how to assign the CCs to each user, and how to multiplex the users within each CC. Different from the downlink, the UE is limited by the maximum transmission power in the uplink, especially for cell edge users since they usually suffer from unfavorable channel conditions. Furthermore, an additional power back-off is needed in the UE power amplifier (PA) with non-contiguous resource allocation in the uplink, which in practice means a reduction of the UE maximum transmission power [25]. The UE transmission power constraint together with the additional power back-off required with non-contiguous resource allocation might counterbalance the gain brought by multi-CC and/or dual-cluster transmission and even results in a performance loss as compared to the case without CA where the SC-FDMA properties of the transmitted signals are maintained (single-CC and singlecluster transmission). Therefore, the selection of UEs to operate with uplink CA has to be carefully considered. The CC selection for uplink CA has been studied in [22] for the case of intra-band contiguous $\mathrm{CA}$ and singlecluster transmission. In this paper, we extend the work of [22] to design efficient radio resource management (RRM) algorithms for uplink CA for cases of both intraband contiguous $\mathrm{CA}$ and inter-band non-contiguous $\mathrm{CA}$, as well as the support for multi-cluster transmission. Our main contributions are as follows:

1) We first focus on the derivation of a simple yet effective pathloss-threshold-based CC configuration algorithm which can be applied to both intra-band $\mathrm{CA}$ and inter-band CA. The proposed algorithm takes some of the key uplink issues into considerations, such as uplink power control and power back-off requirements in the UE PA with non-contiguous resource allocation.

2) To support multi-cluster transmission with in a CC, an extended bandwidth-expansion-based packet scheduling algorithm is proposed based on the work in $[23,26]$. It aims at optimizing the resource allocation with low computation complexity.

3) We present an extensive performance analysis under realistic conditions. Due to the complexity of the system model and the various RRM elements involved, the corresponding performance is best evaluated via advanced system-level simulations under realistic multi-cell, multi-user conditions with dynamic birth-death traffic, using commonly accepted stochastic models and accurate representation of the many mechanisms that influence on the performance.
The rest of the paper is organized as follows: Section 2 outlines the system model of RRM framework for LTEAdvanced, with special attention on multi-cluster transmission, UE power back-off model, and the uplink power control. The proposed CC configuration and packet scheduling algorithms are described in Section 3. Section 4 introduces the simulation methodology and main assumptions. Simulation results and performance analysis are presented in Section 5. Finally, some conclusions are drawn in Section 6.

\section{System model}

In this study, it is assumed that eNodeB (eNB) antennas are collocated and have the same beam patterns for the CCs (also known as CA scenario 1 in the 3rd Generation Partnership Project (3GPP)). This would be a typical scenario for intra-band CA when the CCs are within the same band or different bands but with modest frequency separation, providing nearly the same coverage on all CCs. The RRM framework for multi-CC LTE-Advanced system is illustrated in Figure 1. The layer-3 CC assignment module in the eNB configures one or multiple CCs for each user based on the quality-of-service (QoS) requirements, terminal capability, and CC deployment scenario, etc. Separate RRM blocks such as independent link adaption, packet scheduling, and hybrid automatic repeat request (HARQ) operate independently on each CC in coherence with LTE Rel'8 assumptions [5]. The channel state information (CSI) is extracted from sounding reference signals (SRS) sent by the UE and is used for packet scheduling and link adaptation. As a user may be assigned on multiple CCs, the scheduler in each CC may need to exchange the scheduling information across different CCs in order to achieve better performance [14]. Since the UEs are limited by the transmission power, uplink power control is also an important issue. It is worth mentioning that the algorithms for admission control, CC assignment, and packet scheduling are not part of the standard but are vendor specific.

\subsection{Types of CA and UE transceiver architecture}

Three types of CA have been defined by 3GPP [2]: 1) intra-band $\mathrm{CA}$ with contiguous $\mathrm{CCs}, 2$ ) intra-band $\mathrm{CA}$ with non-contiguous CCs, and 3) inter-band CA with non-contiguous CCs. For the radio frequency (RF) aspects of UEs supporting CA, two options are considered for the baseline UE transceiver architecture, illustrated in Figure 2 [27].

a) Single RF front-end with single wideband analog-todigital converter (ADC) and dual base band (BB) processor

b) Dual RF front-ends with dual narrowband ADCs and dual BBs 


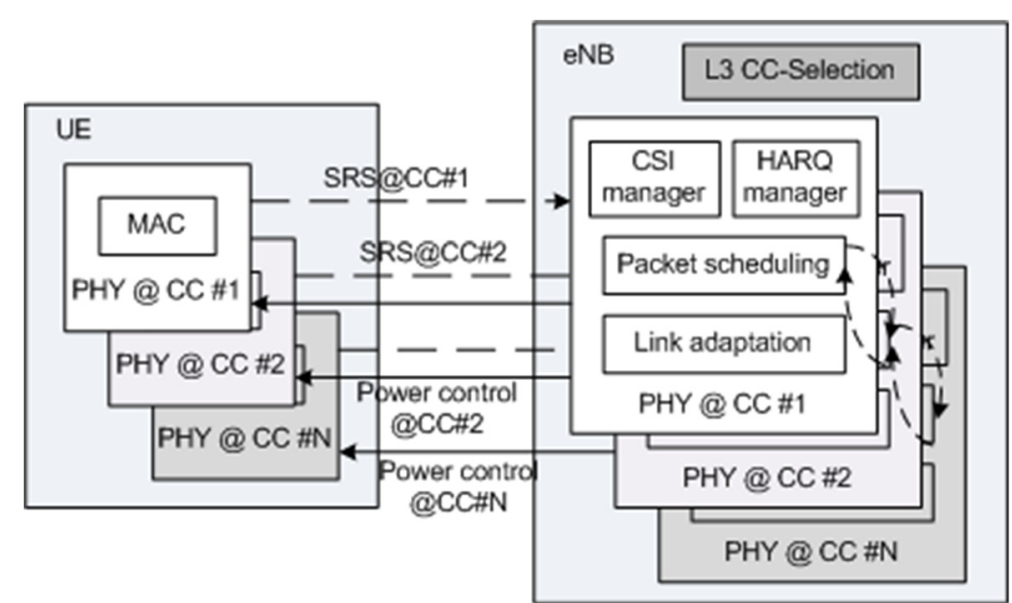

Figure 1 RRM framework of a multi-component carrier LTE-Advanced system.
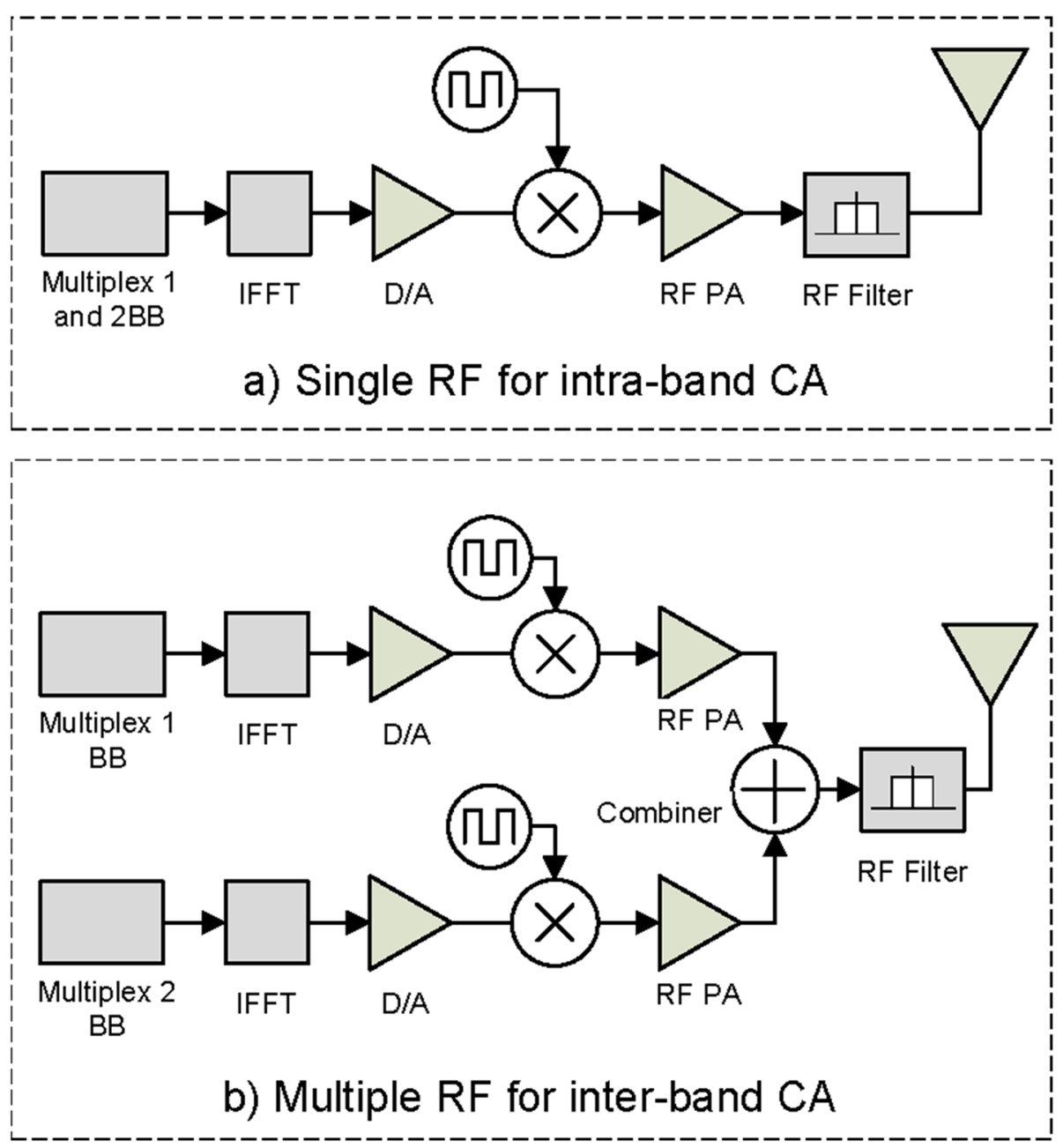

Figure 2 UE transceiver architecture for supporting CA. (a) Single RF for intra-band CA; (b) multiple RF for inter-band CA. 
Option a) is only applicable for intra-band CA with contiguous $\mathrm{CCs}$, because it cannot filter undesired frequency bands between the non-contiguous CCs. The advantage of option a) is keeping the UE transceiver complexity low. For cases of non-contiguous CA, only option b) is applicable at the expense of increased complexity. In this paper, we focus on intra-band CA with contiguous CCs and inter-band CA with non-contiguous CCs. The default UE transceiver architectures for the two considered CA scenarios are therefore option a) and option b), respectively.

\subsection{Multi-cluster transmission in uplink}

In SC-FDMA, UEs in principle can only be scheduled on one set of continuous sub-carriers, which allows SCFDMA to reach very low signal CM but with less scheduling flexibility compared to OFDMA. Multi-cluster transmission has been introduced in Rel'10 to further improve the spectral efficiency by allowing UEs to be scheduled on non-contiguous sub-carriers in the uplink. The minimum resource allocation unit in multi-cluster scheduling is a sub-band, which consists of an integer number of physical resource blocks $\left(\mathrm{PRBs}^{\mathrm{a}}\right)$. Several contiguous sub-bands can be seen as a cluster, and a UE can be allocated to multiple clusters not adjacent to each other within one CC. Therefore, multi-cluster transmission has higher scheduling flexibility compared to SCFDMA while keeping the power back-off requirements at the UE at a reasonable level. Though not considered in this study, it is worth mentioning that the scheduling flexibility of multi-cluster transmission is particular beneficial when combined with non-perfectly overlapping multi-user multiple-input and multiple-output (MU-MIMO) [28]. The three different multiple access schemes are illustrated in Figure 3. A maximum of two clusters within each CC are supported in Rel'10. The sub-band size is a fixed value and depends on the uplink system bandwidth [6]. In our study, the sub-band size corresponds to two PRBs.

\subsection{Maximum power reduction for non-contiguous allocation}

With a single RF front-end at the UE side (e.g., UEs supporting intra-band CA), the UE may be allocated non-contiguous resources when transmitting over multiple CCs, thus the single carrier property of SCFDMA is no longer preserved. The increased $\mathrm{CM}$ of the transmitted signal with non-contiguous resource allocation, and the need to still fulfill the in-band and out-band emission masks [29] require the UE PA to operate with an additional power back-off [27-29]. Furthermore, the multi-cluster transmission within a $\mathrm{CC}$ imposes more stringent linearity requirements on the PA. The exact value of the required power backoff is hard to determine as it is UE implementation specific and is affected by many aspects of the specific uplink resource allocation such as modulation and coding scheme (MCS), number of clusters, size of clusters, frequency separation between clusters, distance from the edge of the first/last cluster to the left/right hand edge of the first/last CC, etc. [30,31]. In this study, we adopt the maximum power reduction (MPR) mask equation proposed by 3GPP [25], though in practice the proposed MPR mask only represents an upper bound of the required power backoff. The MPR value (in $\mathrm{dB}$ ) is determined solely based on the ratio between the allocated and the available bandwidth aggregated from the assigned CCs. For multi-CC transmission, the estimated MPR mask is calculated as:

$$
P_{\mathrm{MPR}}^{\mathrm{CA}}=\left\{\begin{aligned}
8.2, & 0 \quad<A<0.025 \\
9.2-40 A, & 0.025<A \leq 0.05 \\
8-16 A, & 0.05<A \leq 0.25 \\
4.83-3.33 A, & 0.25<A \leq 0.4 \\
3.83-0.83 A, & 0.4<A \leq 1
\end{aligned}\right.
$$

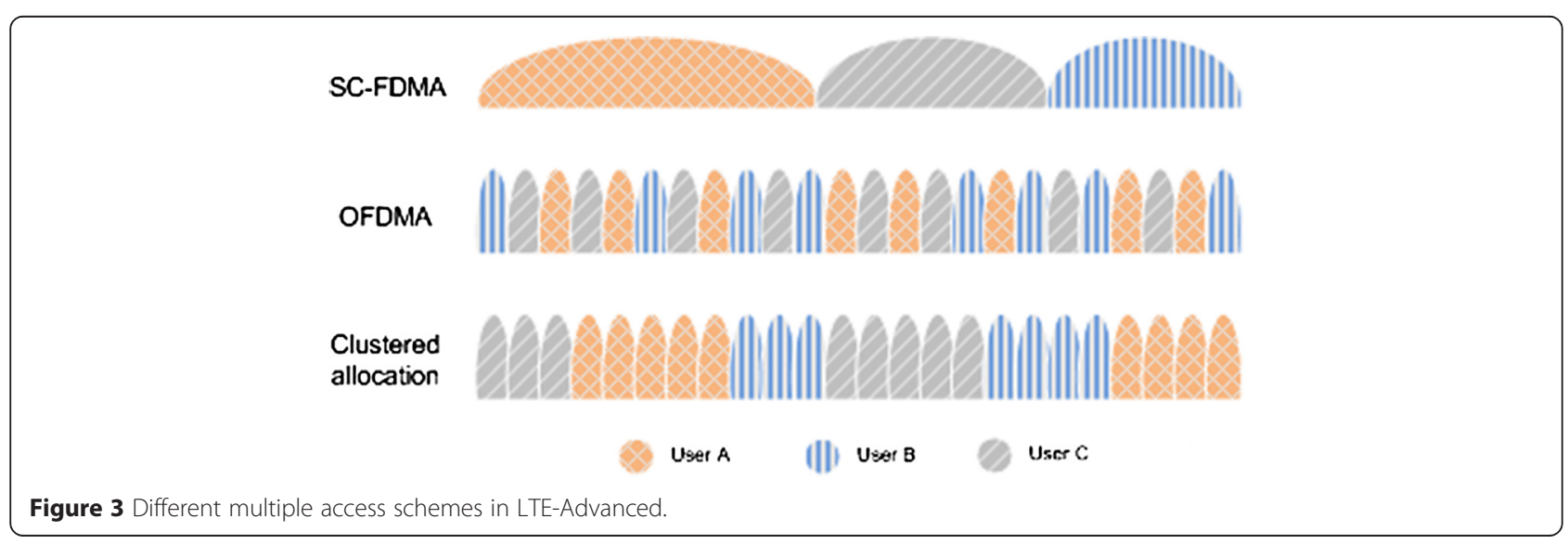


In case of single $\mathrm{CC}$ with multi-cluster transmission, the estimated MPR mask is given by:

$$
P_{\mathrm{MPR}}^{\mathrm{SC}}=\left\{\begin{array}{rll}
8-10.2 A, & 0 & <A \leq 0.33 \\
5.67-3.07 A, & 0.33<A \leq 0.77 \\
3.31, & 0.77<A \leq 1
\end{array}\right.
$$

where $A=N_{\text {PRB_alloc }} / N_{\text {PRB_agg }}$ is the ratio between the number of allocated PRBs (N NRB_alloc $_{\text {) }}$ and total available PRBs over all assigned CCs ( $\left.\mathrm{N}_{\text {PRB_agg }}\right)$.

With dual RF front-ends at the UE side (e.g., UEs supporting inter-band CA), each carrier is amplified by a separate PA and the two carriers are added up in the combiner as illustrated in Figure 2. In this case, the power back-off issue is less important if two PA are sufficiently isolated from each other. Note that the use of a combiner results in insertion loss of at least $2 \mathrm{~dB}$ [27]. However, if multi-cluster transmission is enabled within each CC, power back-off is still needed in the UE PA with the MPR value specified in Equation 2. It is worth mentioning that the power back-off model for inter-band CA with multi-cluster transmission is built from existing models using simplified assumptions. Table 1 summarizes the required power back-off values for different CA types and uplink resource allocation schemes.

\subsection{Uplink power control}

As the UE is limited by the maximum transmission power, the uplink power control is an important issue. It consists of open loop power control and closed loop power control. The open loop power control aims at compensating for slow variations of channel conditions, i.e., pathloss and shadowing. The closed loop power control targets at further adjusting the UE's transmission power to optimize the system performance.

In Rel'10, independent power control is applied in each CC so that Rel'8 power control formula can also be reused in case of $\mathrm{CA}$. The estimated transmission power on each assigned CC depends on the allocated bandwidth and on the $\mathrm{CC}$ specific power control parameters. When user $i$ is scheduled for transmission on CC $k$, the

Table 1 Required power back-off values for different types of CA and uplink resource allocation schemes

\begin{tabular}{lll}
\hline CA type & $\begin{array}{l}\text { Resource } \\
\text { allocation }\end{array}$ & $\begin{array}{l}\text { Required power } \\
\text { back-off (dB) }\end{array}$ \\
\hline $\begin{array}{l}\text { Intra-band CA with } \\
\text { one RF front-end }\end{array}$ & Multi-CC transmission & $P_{M P R}^{C A}$ \\
& Single-CC with multi-cluster & $P_{M P R}^{S C}$ \\
& Single-CC with single-cluster & 0 \\
Inter-band CA with & Multi-CC with single-cluster & 2 \\
dual RF front-ends & Multi-CC with multi-cluster & $P_{M P R}^{S C}+2$ \\
\hline
\end{tabular}

estimated transmission power $\tilde{P}_{i, k}(t)$, expressed in $\mathrm{dBm}$, is calculated as [32]:

$$
\tilde{P}_{i, k}(t)=10 \log _{10} M_{i, k}(t)+P_{0, k}(t)+f\left(\Delta_{i, k}(t)\right)+\alpha_{k} \cdot L_{i, k}
$$

where $M_{i, k}(t)$ is the number of PRBs allocated to user $i$ on CC $k, P_{0, k}(t)$ is the normalized power density on CC $k, \alpha_{k}$ is the pathloss compensation factor on CC $k, L_{i, k}$ is the measured pathloss between user $i$ and its serving base station on CC $k$, and $f\left(\Delta_{i, k}(t)\right)$ is the closed loop power control correction for user $i$ on CC $k$. More details on the uplink power control formula for LTEAdvanced with CA can be found in [7].

Previous studies [33,34] have shown that the system performance in terms of coverage (5th percentile user throughput) and cell throughput is highly dependent on the setting of $P_{0, k}$ and $\alpha_{k}$. Load adaptive power control (LAPC) is proposed in [34] to dynamically adjust the users' power spectral density $P_{0, k}$. If the optimization goal is to maximize the coverage, the 5 th percentile cell edge users should transmit with the maximum transmission power. Then, the power spectral density is updated periodically according to the following equation:

$$
P_{0, k}(t)+f\left(\Delta_{i, k}(t)\right)=P_{\max }-10 \log _{10} M_{k}(t)-\alpha_{k} \cdot L_{95 \%, k}
$$

where $P_{\max }$ is the maximum UE transmission power in $\mathrm{dBm}, M_{k}(t)$ is the estimated average number of allocated PRBs allocated to each user on CC $k$, and $L_{95} \%$ is the estimated 95th percentile user pathloss in the corresponding cell on CC $k$. For simplicity, $\bar{M}_{k}(t)$ is approximated as the total number of available PRBs divided by the number of UEs served by the corresponding cell.

Because power control is performed independently within each $\mathrm{CC}$, the aggregated transmission power allocated over all CCs may exceed the maximum UE transmission power constraint even if the individual transmission power within each $\mathrm{CC}$ is below the limit. In this case, the UE needs to scale down the transmission power with different priorities according to the channel type [35]. In our study, the reduction of transmission power for user $i$ on each assigned CC follows the same ratio. The actual transmission power $P_{i, k}(t)$ of user $i$ on CC $k$ then becomes:

$$
P_{i, k}(t)=\tilde{P}_{i, k}(t)-\max \left\{0,10 \log _{10}\left(\sum_{k=1}^{N} 10^{\tilde{P}_{i, k}(t) / 10}\right)-\left(P_{\max }-P_{\text {backoff }}\right)\right\}
$$

where $N$ is the number of CCs assigned to user $i$, and $P_{\text {backoff }}$ is the required power back-off with the values listed in Table 1. 


\section{Component carrier configuration and packet scheduling}

The two main RRM functionalities that determine the radio resource allocation among the users in the system are the layer $3 \mathrm{CC}$ configuration which selects a $\mathrm{CC}$ set for each user, and the layer 2 packet scheduling which allocates the resources among the admitted users on each CC. Hence, these two RRM functionalities are also the ones that impact the most on the performance of uplink CA. The proposed algorithms for these two sets of RRM algorithms are therefore motivated and outlined in the following.

\subsection{Component carrier configuration}

In a multi-CC LTE-Advanced system, both legacy and LTE-Advanced users may co-exist. The legacy Rel'8 users naturally can only be assigned on a single CC. With intra-band $\mathrm{CA}$, the radio channel characteristics of different CCs are more or less the same. For optimal system performance, it is desirable to distribute the load equally on each CC. So, a simple yet effective load balancing scheme is applied for Rel'8 users, which assigns the CC with the least load (e.g. the number of users). With inter-band CA, the radio channel characteristics can be different at different frequency carriers. The study in [10] indicates that the CC selection should take both traffic load and radio channel characteristics into considerations. Therefore, with inter-band CA, celledge Rel'8 users (e.g., 5th percentile in pathloss distribution) are assigned to the $\mathrm{CC}$ with better coverage (i.e., low-frequency carrier) to improve the coverage performance, while other Rel'8 users are assigned to the $\mathrm{CC}$ with the least number of users to balance the load on each CC.

LTE-Advanced users can be assigned on multiple CCs. A CC is defined as its primary cell (PCell) and can only be changed via handover. Different users may not necessarily use the same CC as their PCell. If more than one $\mathrm{CC}$ is configured, the additional CCs are denoted as secondary cells (SCells). The SCells can be activated/deactivated by explicit signaling from the eNodeB, as well as based on appositely configured timers [36].

LTE-Advanced users configured with multiple CCs have the possibility to transmit on multiple CCs using the corresponding power control settings in each $\mathrm{CC}$, therefore requiring higher transmission power as compared to the case with single CC configuration. Configuring LTE-Advanced users with multiple CCs and/or dual-cluster transmission in principle only makes sense if its total transmission power does not exceed the maximum UE power capability. Otherwise, they do not have sufficient power to exploit the increased transmission bandwidth and scheduling flexibility. For an LTE-
Advanced user $i$ assigned on $N$ CCs, the total transmission power is calculated as:

$$
P_{i}(t)=10 \log _{10}\left(\sum_{k=1}^{N} 10^{P_{i, k}(t) / 10}\right) \leq P_{\max }-P_{\text {backoff }}
$$

Substituting Equations 3 and 4 into Equation 6 and assuming $M_{i, k}(t)=M_{k}(t)$, we obtain:

$$
\sum_{k=1}^{N} 10^{\alpha_{k}\left(L_{i, k}-L_{95 \%, k}\right)} \leq 10^{\frac{P_{\text {backoff }}}{10}}
$$

where $P_{\text {backoff }}$ is the expected power back-off with noncontiguous resource allocation. So with uplink CA, Equation 7 can be used to estimate whether the UE has enough transmission power to manage transmissions on multiple CCs simultaneously, assuming LAPC is applied. For the case of intra-band CA, it is often assumed that $\alpha_{k}$ and $L_{95} \%$ are the same on each CC $k$, then Equation 7 can be further simplified into:

$$
L_{i} \leq L_{95 \%}-\frac{10 \log _{10} N+P_{\text {backoff }}}{\alpha}
$$

Based on the derived pathloss threshold, we propose a CC configuration algorithm for uplink CA. The idea is to distinguish between power-limited and non-powerlimited users. LTE-Advanced users whose pathloss satisfies Equation 7 are considered to be non-power-limited and can be assigned on multiple CCs with dual-cluster transmission. Otherwise, they are considered to be power-limited and are only assigned on one $\mathrm{CC}$ with single-cluster transmission. The selection of the CC for power-limited LTE-Advanced users is the same as for Rel'8 users. By separating the power-limited and nonpower-limited LTE-Advanced users, cell-edge LTEAdvanced users will not experience any performance loss with non-contiguous resource allocation, while nonpower-limited LTE-Advanced users can benefit from increased transmission bandwidth and scheduling flexibility with $\mathrm{CA}$ and multi-cluster transmission. It is worth mentioning that with non-contiguous resource allocation, the required power back-off specified in Equation 1 is not dependent on the number of clusters, but rather depends on the ratio between the allocated and total available bandwidth [30]. Therefore, for non-powerlimited LTE-Advanced users, it is always beneficial to use dual-cluster transmission in combination with CA in order to increase the scheduling flexibility. The proposed $\mathrm{CC}$ and multi-cluster configuration algorithm is illustrated in Figure 4.

\subsection{Bandwidth allocation and packet scheduling}

After the CC and cluster assignment for each user, the layer 2 time and frequency domain packet scheduler 


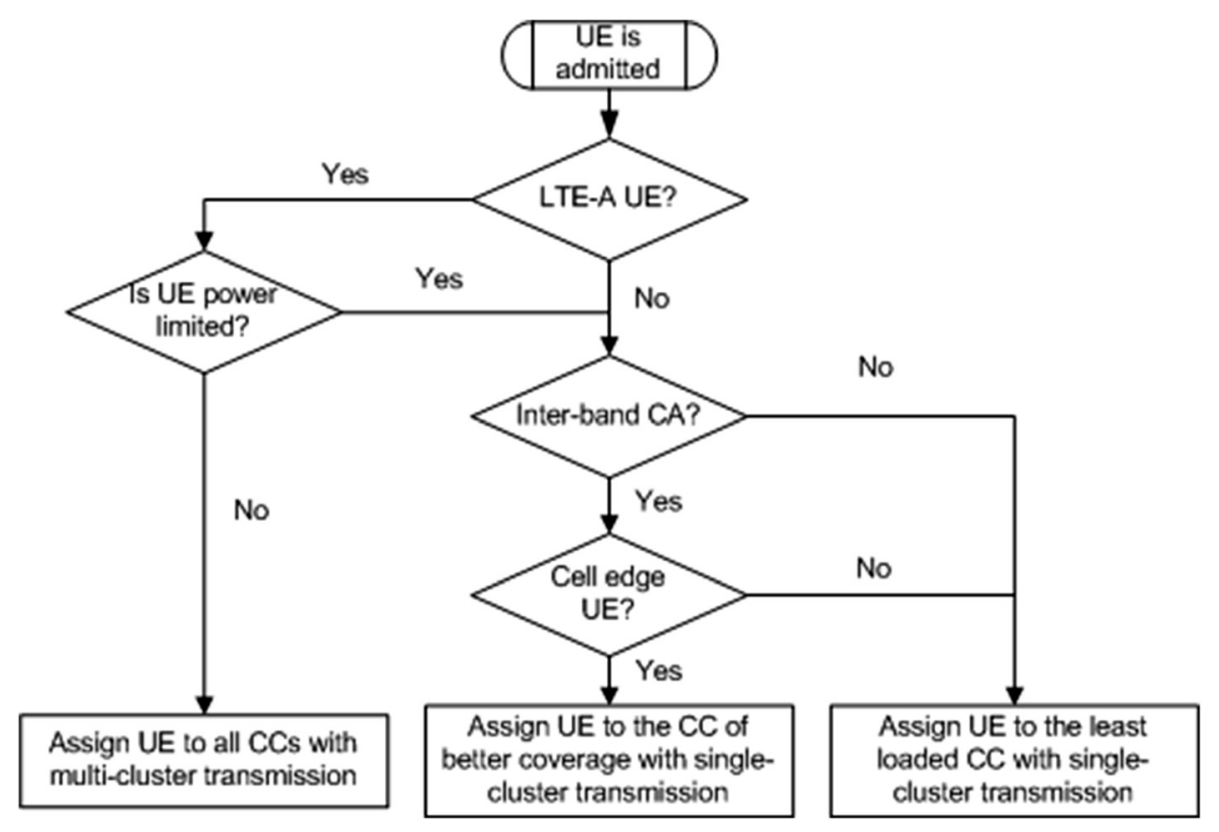

Figure 4 Proposed CC and multi-cluster configuration algorithm in uplink LTE-Advanced.

allocates the resources in terms of PRBs among the admitted users on each CC according to certain constraints with the objective to improve the spectral efficiency while maintaining fairness among scheduled users. The use of sounding reference signals (SRS) allows for uplink link adaptation and channel-aware frequency domain packet scheduling [7]. The eNB may configure the SRS patterns on each carrier depending on the accuracy of uplink SRS measurements.

In [26], the authors proposed an adaptive transmission bandwidth (ATB)-based packet scheduling algorithm for SC-FDMA with single-cluster transmission in uplink, which tightly couples the bandwidth allocation and the packet scheduling together to exploit the bandwidth flexibility. In this study, we extend the ATB algorithm so that it is catered to support multi-cluster transmission [23]. With multi-cluster transmission, the allocated PRBs for a user can be non-contiguous subject to the constraint of not exceeding the maximum number of configured clusters, which is determined according to the algorithm depicted in Figure 4. The basic idea behind the proposed scheduling algorithm is to produce an allocation table which closely follows the envelope of the UEs' scheduling metrics.

We assume that packet scheduling is performed in each CC. The well-known proportional fair (PF) scheduler is applied. The problem with baseline PF scheduler is that it will allocate more resources to the users connected to multiple CCs (with CA) than users without CA, resulting in an unfair resource allocation. Therefore, cross-carrier PF scheduling across multiple CCs is recommended when calculating the scheduling metric in each CC [14]. In cross-carrier PF scheduler, the denominator of the PF metric is calculated as the sum of the average scheduled throughput over all CCs where the user has been assigned and scheduled in the past. By applying this modification, the scheduler can distribute the radio resources among the users more fairly. It simply requires information exchange on the average scheduled throughput across different CCs. The scheduling metric of user $i$ at sub-band $j$ on CC $k$, denoted as $M_{i, j, k}$, is therefore calculated as:

$$
M_{i, j, k}(t)=\frac{R_{i, j, k}(t)}{\sum_{k=1}^{N} R_{i, k}(t)}
$$

where $R_{i, j, k}(t)$ is the estimated throughput of user $i$ at sub-band $j$ on CC $k$, and $R_{i, k}(t)$ is the exponentially filtered average throughput of user i on CC $k$.

At each scheduling instance, the scheduler in each CC first generates a scheduling matrix of each user on each sub-band. Then, the scheduler selects a UE and the corresponding sub-band with the highest scheduling metric and checks the power constraint of that UE with Equation 3. If the selected UE exceeds its maximum transmission power capability with the new resource allocation, the scheduler removes that UE from the candidate list and reselects the $\mathrm{UE}$ and the sub-band from the scheduling matrix. After the power constraint check, the scheduler checks the number of allocated clusters of the selected UE. If the new resource allocation causes the number of allocated clusters to exceed the maximum number of configured clusters (i.e., singlecluster for Rel'8 and power-limited LTE-A users, and dual- 
cluster for non-power-limited LTE-A users), the current sub-band is disabled for that UE and the algorithm restarts from the beginning. Otherwise, the scheduler allocates the current sub-band to that UE and expands its transmission bandwidth to the adjacent sub-bands on both sides until either another UE has a higher scheduling metric or the maximum transmission power of that UE is exceeded. Details of the bandwidth expansion procedure can be found in [26]. Then, the algorithm restarts from the beginning and continues the loop until either all UEs have been scheduled or there are no resources left. A detailed description of the proposed multi-cluster scheduling algorithm is illustrated in Figure 5 [23].

\section{Simulation assumptions}

The performance of the proposed algorithms is evaluated in a quasi-static uplink multi-cell system level simulator that follows the LTE specifications defined in [37], including detailed modeling of major RRM functionalities such as layer $3 \mathrm{CC}$ configuration, layer 2 packet scheduling, HARQ, link adaptation, and uplink power control. The simulation scenario is 3GPP macro-cell case number 1 with seven sites and three sectors per site using the wrap-around technique. A directional 3D antenna pattern with default $15^{\circ}$ down-tilt is modeled for the macro cells. The ITU defined geometrical channel model is applied, where UE to macro links follow the urban macro model (UMa), which includes separate models for line-of-sight (LOS) and non-LOS (NLOS) [38]. Selection between the LOS and NLOS model is random for each link, where the probabilities for selecting LOS or NLOS vary with the distance between the UE and eNB. Small-scale fading (also known as fast fading) is modeled according to the commonly accepted stochastic typical urban model. It is assumed that distance-dependent path loss and shadowing are maintained constant for each UE, but fast fading is updated every TTI independently on each CC based on the ITU typical urban power delay profile and UEs' speed. For intra-band CA, two CCs each with $20 \mathrm{MHz}$ bandwidth are deployed at $2 \mathrm{GHz}$. For inter-band CA, two CCs each with $20 \mathrm{MHz}$ bandwidth are deployed at 1.8 and $2.6 \mathrm{GHz}$, respectively. Up to two clusters are supported in each CC for uplink multi-cluster transmission. The SRS resolution is two PRBs and the SRS period is set to be ten subframes. Imperfect SRS measurements are assumed. The measurement error is a function of the experienced signal-to-interference-plus-noise ratio (SINR) and sounding bandwidth. The link to system level mapping is based on the actual value interface (AVI) method [39], including channel estimation error with uplink clustered transmission which is modeled by modifying the experienced SINR before it is used for AVI mapping. Both full buffer and bursty traffic models are considered. In full-buffer traffic model, we assume each sector has a fixed number of UEs with full buffer traffic. For bursty traffic model, a dynamic birth-death traffic model is applied for generating user calls, where call arrival is according to a Poisson process with arrival rate $\lambda$ per sector. Each call has a finite payload size of $B=2$ Mbits.

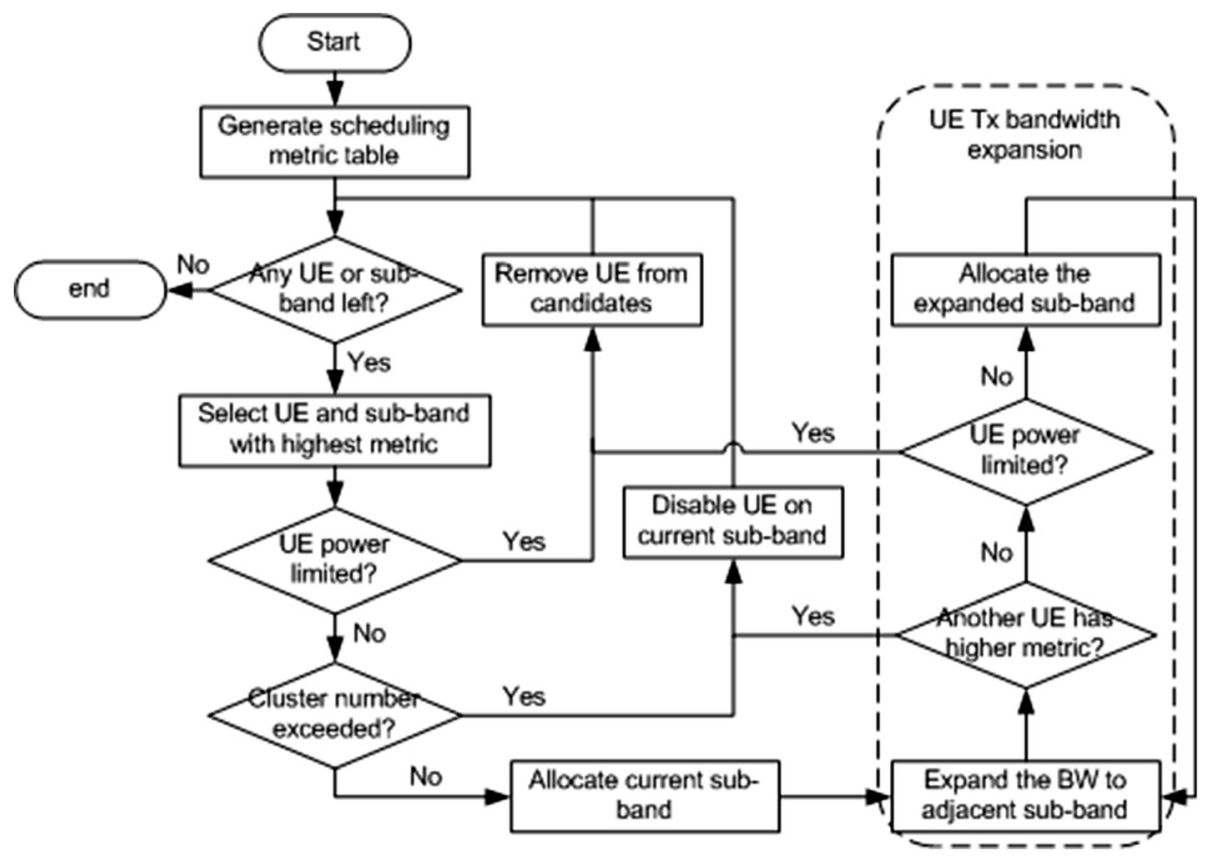

Figure 5 Proposed uplink multi-cluster scheduling algorithm in LTE-Advanced. 
Once the payload has been successfully transmitted by the UE, the call is terminated and the UE is removed from the simulation. Thus, the average offered load per sector equals $\lambda \times B$. Joint proportional fair scheduling in frequency domain is used together with multi-cluster bandwidth allocation. Independent LAPC is enabled on each CC to dynamically adjust the UE power spectral density based on the variable load conditions. The optimization goal is to maximize the 5th percentile cell edge user throughput. The average UE power back-off $P_{\text {backoff }}$ with non-contiguous resource allocation is set to be $6 \mathrm{~dB}$ (close to worst-case assumption considering the power back-off model in Equation 1). Table 2 summarizes the main parameter settings used in the systemlevel simulations.

\section{Simulation results and analysis}

\subsection{Multi-cluster transmission gain in uplink}

We start our analysis by first looking at the scenario with a fixed number of UEs per sector and full-buffer traffic model. Only one CC is configured with $10 \mathrm{MHz} @ 2 \mathrm{GHz}$ in this scenario. Figure 6 shows the dual-cluster scheduling gain over single-cluster scheduling and the average number of scheduled clusters per UE versus the different number of UEs per macro sector, respectively. It is shown that the dual-cluster scheduling gain is closely correlated to the average number of scheduled clusters per UE. Specifically, the dual-cluster scheduling gain increases as the number of UEs increases until reaching the maximum value, i.e., $16 \%$ gain at six UEs per macro sector in our case. Then, the gain gradually decreases as the number of UEs increases. That is because when the number of UEs per macro sector is low (e.g., less than six), dual-cluster scheduling users have more chance to exploit the increased frequency diversity order as compared to single-cluster transmission. However, when the number of UEs per macro sector is high (e.g., larger than six), the probability of a user being scheduled on multiple clusters decreases, therefore the gain brought by dual-cluster scheduling decreases.

\subsection{Effectiveness of the proposed CC configuration algorithm}

Next, we evaluate the effectiveness of the derived pathloss threshold in Equation 8 against other pathloss thresholds with bursty traffic model. Two contiguous CCs each with $20 \mathrm{MHz}$ bandwidth are configured for intra-band $\mathrm{CA}$ in this scenario. Figure 7 shows the 5th percentile and 50th percentile user throughput versus the different pathloss thresholds under different traffic loads (5 and $20 \mathrm{Mbps}$ offered loads correspond to approximately $20 \%$ and approximately $70 \%$ PRB utilization, respectively). Here, we assume $100 \%$ LTE-Advanced UEs. The 5th percentile user throughput stays almost constant when the pathloss threshold is smaller than a certain value (approximately $109 \mathrm{~dB}$ ). After that point, the 5th percentile user throughput decreases as the pathloss threshold increases. For the 50th percentile user throughput, it increases as the threshold increases until the maximum value is reached also around $109 \mathrm{~dB}$. Then, the throughput decreases with further increase of the pathloss threshold. The reasons are as follows: if the pathloss threshold is set to be low, most UEs, including power-limited and some non-power-limited UEs are only assigned on one $\mathrm{CC}$ with single-cluster transmission. As a result, those non-power-limited UEs cannot benefit from the advantages of transmission bandwidth expansion by using CA and increased scheduling flexibility with dual-cluster transmission. Therefore, small value of the threshold would decrease the 50th percentile user throughput. The 5th percentile user throughput remains steady as they are assigned only on one CC. On the other hand, if the pathloss threshold is set to be high, not only non-power-limited but also some powerlimited UEs are assigned on both CCs with dual-cluster transmission. As a result, those power-limited cell edge UEs will experience performance loss from being scheduled over multiple CCs and clusters due to the effect of additional power back-off with non-contiguous resource allocation. Since cell edge users usually utilize the resources with low efficiency due to poor channel conditions, the increase of their activity time in the network worsens the resource utilization of other users. Therefore, higher value of the threshold would decrease both the 5th percentile and 50th percentile user throughput. As a conclusion, the value of pathloss threshold has to be carefully determined in order to optimize the system performance. It is shown from Figure 7 that our derived pathloss threshold works effectively to achieve good performance at both 5th percentile and 50th percentile user throughput under different traffic conditions. Thus, it is robust against variations in the traffic load.

\subsection{Overall performance}

Then, we evaluate the performance of intra-band and inter-band CA with the proposed CA algorithm as compared to the case without CA in a bursty traffic model. The performance with 'blind' CC configuration (i.e. all UEs are configured with uplink $C A$ ) is also included for comparisons. Figure 8 shows the 5 th percentile user throughput versus the offered load with different CA and multi-cluster transmission schemes. As expected, the user throughput decreases as the load in the network increases. For intra-band CA, there is a performance loss if all UEs are configured with CA and/or multi-cluster transmission, as compared to the case without $\mathrm{CA}$ and single-cluster transmission. This is because at the cell edge, UEs usually experience large pathloss and are 
Table 2 Summary of main simulation parameters

\begin{tabular}{|c|c|c|}
\hline Parameters & \multicolumn{2}{|l|}{ Settings } \\
\hline Simulation scenario & \multicolumn{2}{|c|}{ 3GPP macro case number 1} \\
\hline Network layout & \multicolumn{2}{|c|}{ Seven macro sites - three sectors/site - wrap around } \\
\hline \multirow[t]{4}{*}{ Channel model (UMa) [38] ${ }^{\mathrm{a}}$} & LOS pathloss & $22 \log _{10}(d)+28.0+20.0 \log _{10}(f)$, shadow fading $s t d=4 d B, 10 m<d<d_{B P}^{\prime}$ \\
\hline & & $\begin{array}{l}40 \log _{10}(d)+7.8-18 \log _{10}\left(h_{\mathrm{BS}}^{\prime}\right)-18 \log _{10}\left(h_{\mathrm{UT}}^{\prime}\right)+2 \log _{10}(f), \text { shadow fading } \\
\mathrm{std}=4 \mathrm{~dB}, d_{\mathrm{BP}}^{\prime}<d<5,000 \mathrm{~m}\end{array}$ \\
\hline & NLOS pathloss & $\begin{array}{l}161.04-7.1 \log _{10}(W)+7.5 \log _{10}(h)-\left(24.37-3.7\left(h / h_{B S}\right)^{2}\right) \log _{10}\left(h_{B S}\right)+ \\
\left(43.42-3.1 \log _{10}\left(h_{B S}\right)\right)\left(\log _{10}(d)-3\right)+20.0 \log _{10}(f)-\left(3.2\left(\log _{10}\left(11.75 h_{U T}\right)\right)^{2}-4.97\right) \\
\text { shadow fading std }=6 d B, 10 \mathrm{~m}<d<5,000 \mathrm{~m}\end{array}$ \\
\hline & LOS probability & $\min \left(\frac{18}{d}, 1\right)\left(1-\exp \left(-\frac{d}{63}\right)\right)+\exp \left(-\frac{d}{63}\right)$ \\
\hline Inter-site distance & $500 \mathrm{~m}$ & \\
\hline \multirow[t]{2}{*}{ Component carriers } & \multicolumn{2}{|c|}{ Intra-band CA: 2×20 MHz @2 GHz, inter-band CA: 20 MHz@1.8 GHz+20 MHz@2.6GHz } \\
\hline & \multicolumn{2}{|c|}{96 available PRBs for PDSCH per CC } \\
\hline Number of clusters & \multicolumn{2}{|c|}{ Single or dual clusters } \\
\hline PRBs per sub-band & \multicolumn{2}{|l|}{ Two PRBs } \\
\hline Sounding resolution & \multicolumn{2}{|l|}{ Two PRBs } \\
\hline Sounding period & \multicolumn{2}{|l|}{ Ten subframes } \\
\hline Sounding method & \multicolumn{2}{|l|}{ Imperfect SRS } \\
\hline eNode-B receiver & \multicolumn{2}{|l|}{ 4-RX MMSE } \\
\hline Max UE power & \multicolumn{2}{|c|}{200 mW (23 dBm) } \\
\hline \multirow[t]{2}{*}{ UE transceiver } & \multicolumn{2}{|c|}{ Single transceiver for intra-band CA } \\
\hline & \multicolumn{2}{|c|}{ Two separate transceivers for inter-band CA } \\
\hline UE Tx bandwidth & \multicolumn{2}{|c|}{ ATB for multi-cluster } \\
\hline Packet scheduling & \multicolumn{2}{|c|}{ Joint proportional fair } \\
\hline \multirow[t]{2}{*}{ Bursty traffic model } & \multicolumn{2}{|c|}{ Finite buffer with different Poisson arrival rate } \\
\hline & \multicolumn{2}{|c|}{ Fixed payload size of 2 Mbits per UE } \\
\hline Full-buf. traffic model & \multicolumn{2}{|c|}{ Full buffer with fixed number of UEs } \\
\hline UE speed & \multicolumn{2}{|l|}{$3 \mathrm{~km} / \mathrm{h}$} \\
\hline \multirow[t]{3}{*}{ Available MCSs } & \multicolumn{2}{|c|}{$\operatorname{BPSK}(R=1 / 5,1 / 3)$} \\
\hline & \multicolumn{2}{|c|}{$\operatorname{QPSK}(R=1 / 4,1 / 3,1 / 2,2 / 3,3 / 4)$} \\
\hline & \multicolumn{2}{|c|}{16 QAM $(R=1 / 2,2 / 3,3 / 4,5 / 6)$} \\
\hline HARQ & \multicolumn{2}{|c|}{ Synchronous and adaptive with maximum of four transmissions } \\
\hline First Tx BLER target & \multicolumn{2}{|l|}{$20 \%$} \\
\hline Link adaptation & Fast adaptive $n$ & d coding (AMC) \\
\hline Pathloss compensation & $a=0.8$ & \\
\hline 95th percentile user pathloss & 118 dB@1.8GH & $\mathrm{GHz}, 123 \mathrm{~dB} @ 2.6 \mathrm{GHz}$ \\
\hline Power spectral density & Independent $\mathrm{L}$ & CC [34] \\
\hline Average power back-off & $P_{\text {backoff }}=6 \mathrm{~dB}$ & \\
\hline
\end{tabular}

${ }^{a} f$ is the center frequency $(\mathrm{Hz}), h_{\mathrm{BS}}$ and $h_{\mathrm{UT}}$ are the actual antenna heights at the $\mathrm{BS}$ and the $\mathrm{UT}(\mathrm{m}), h_{\mathrm{BS}}^{\prime}$ and $h_{\mathrm{UT}}^{\prime}$ are the effective antenna heights at the BS and the UT $(\mathrm{m}), d$ is the distance $(\mathrm{m}), d_{B P}^{\prime}$ is the break point distance $d_{B P}^{\prime}=4 h_{B S}^{\prime} h_{U T}^{\prime} f / c$ where $c$ is the propagation velocity in free space, $W$ is the street width (m), and $h$ is the average building height $(\mathrm{m})$.

limited by the transmission power. In fact, with LAPC, cell edge UEs (worst 5th percentile UEs) are configured to transmit with the maximum transmission power on the assigned CC. Transmitting over multiple CCs and/or multiple clusters will cause further power reduction due to the effect of additional power back-off with non-contiguous resource allocation, therefore degrading the 5 th percentile UE throughput performance. In the proposed CA algorithm, the power-limited cell edge UEs are only assigned on one CC with single-cluster transmission so that the SC-FDMA properties of the transmitted signals are maintained. Therefore, there is no performance loss as compared to Rel'8 operation. For inter-band CA with single-cluster transmission, the performance is slightly worse as compared to 


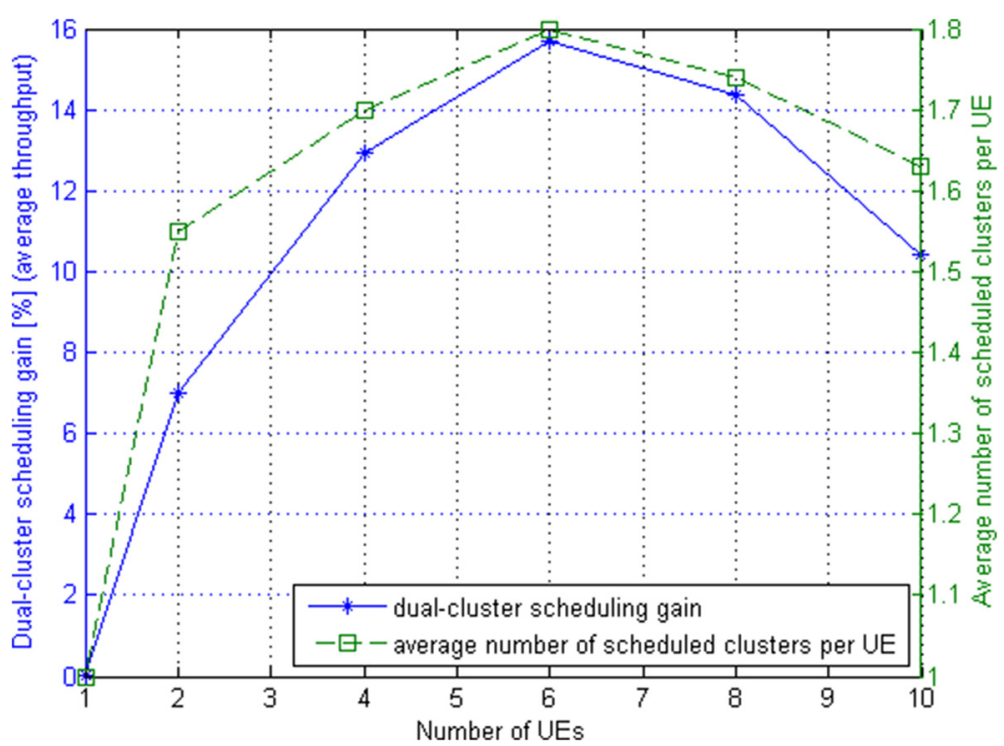

Figure 6 Multi-cluster scheduling gain with full buffer traffic.

the case of intra-band $\mathrm{CA}$, due to the 2-dB insertion loss in the combiner with two RF front-ends and the higher frequency band used for inter-band CA. For inter-band $\mathrm{CA}$ with dual-cluster transmission, the dual-cluster transmission should be avoided for cell edge users as it will cause additional power back-off similar to the case of intra-band CA.

Figure 9 shows the 50th percentile user throughput versus the offered load with different $\mathrm{CA}$ and multicluster transmission schemes. For intra-band $\mathrm{CA}$, the

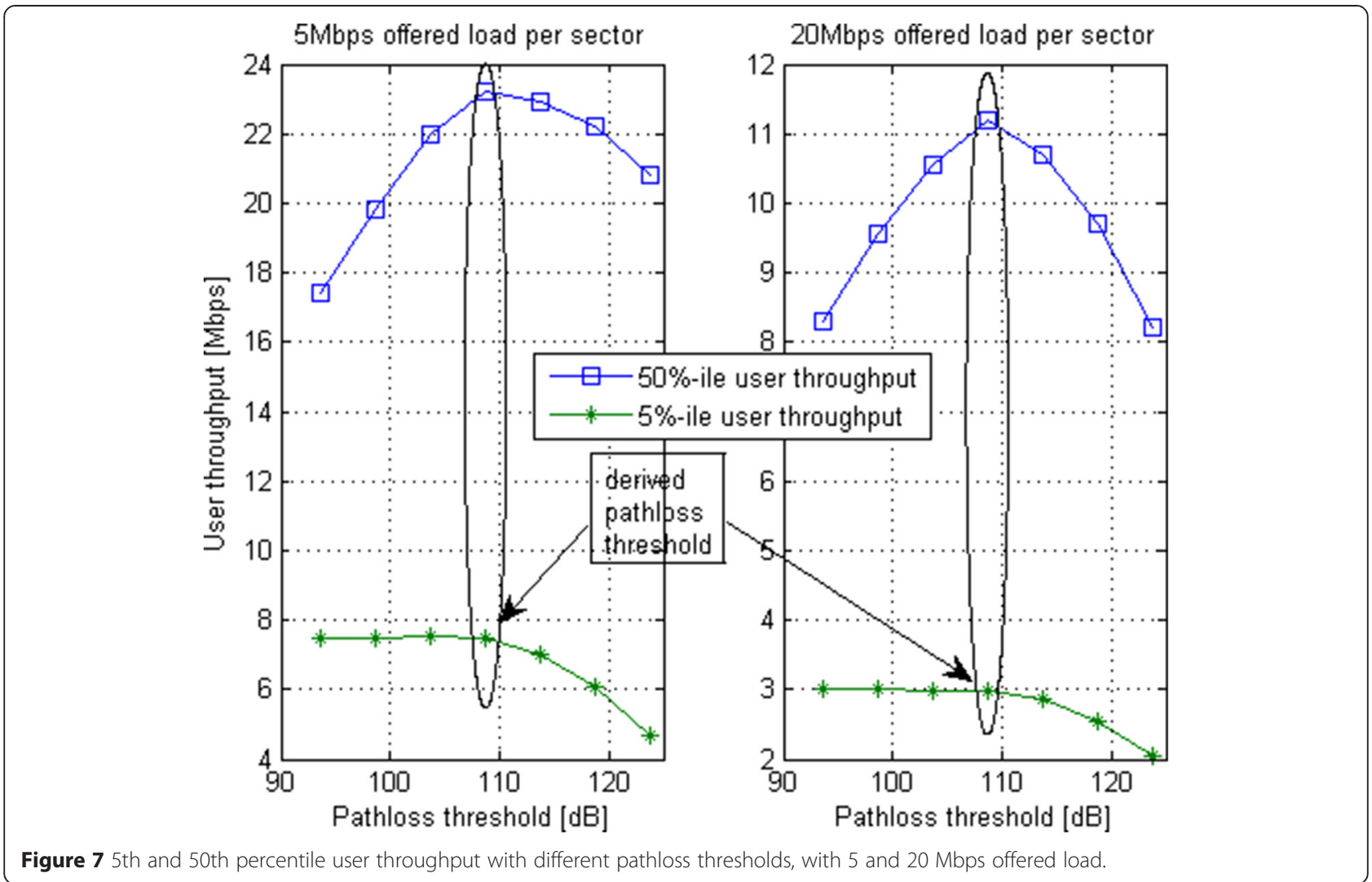



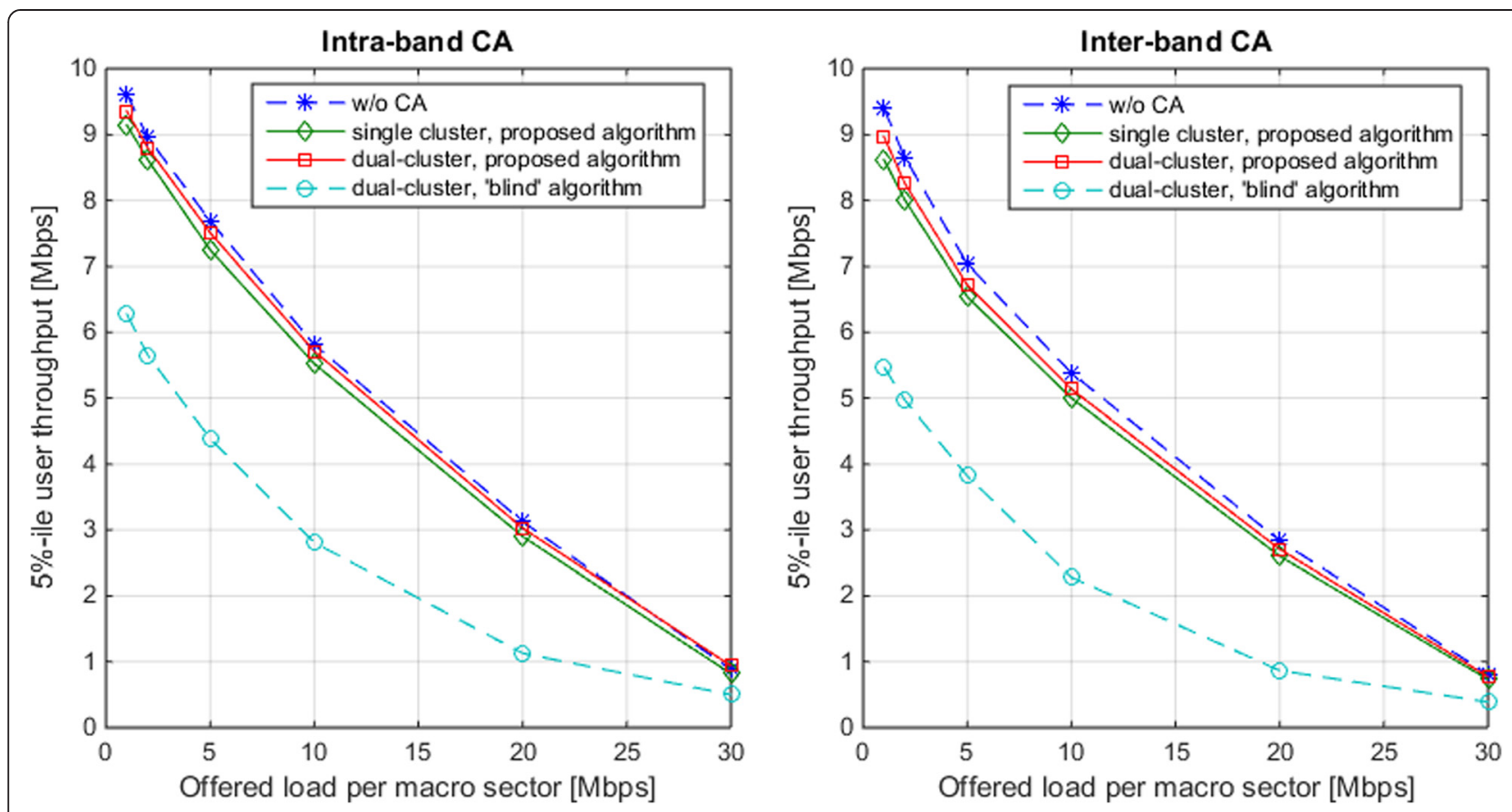

Figure 8 5th percentile user throughput under different traffic loads in different scenarios.

user throughput with the proposed CA algorithm is significantly higher than that with 'blind' CA configuration and the case without CA. Also, it is observed that dualcluster transmission can further increase the user throughput. That is because with 'blind' CA configuration, cell edge UEs will stay in the system for longer time due to the poor performance as shown in Figure 8, therefore occupying more resources. In the proposed CA algorithm, only the non-power-limited UEs are assigned on multiple CCs with dual-cluster transmission so that they can benefit from the advantages of CA (i.e., increased transmission bandwidth with multiple $\mathrm{CCs}$ )
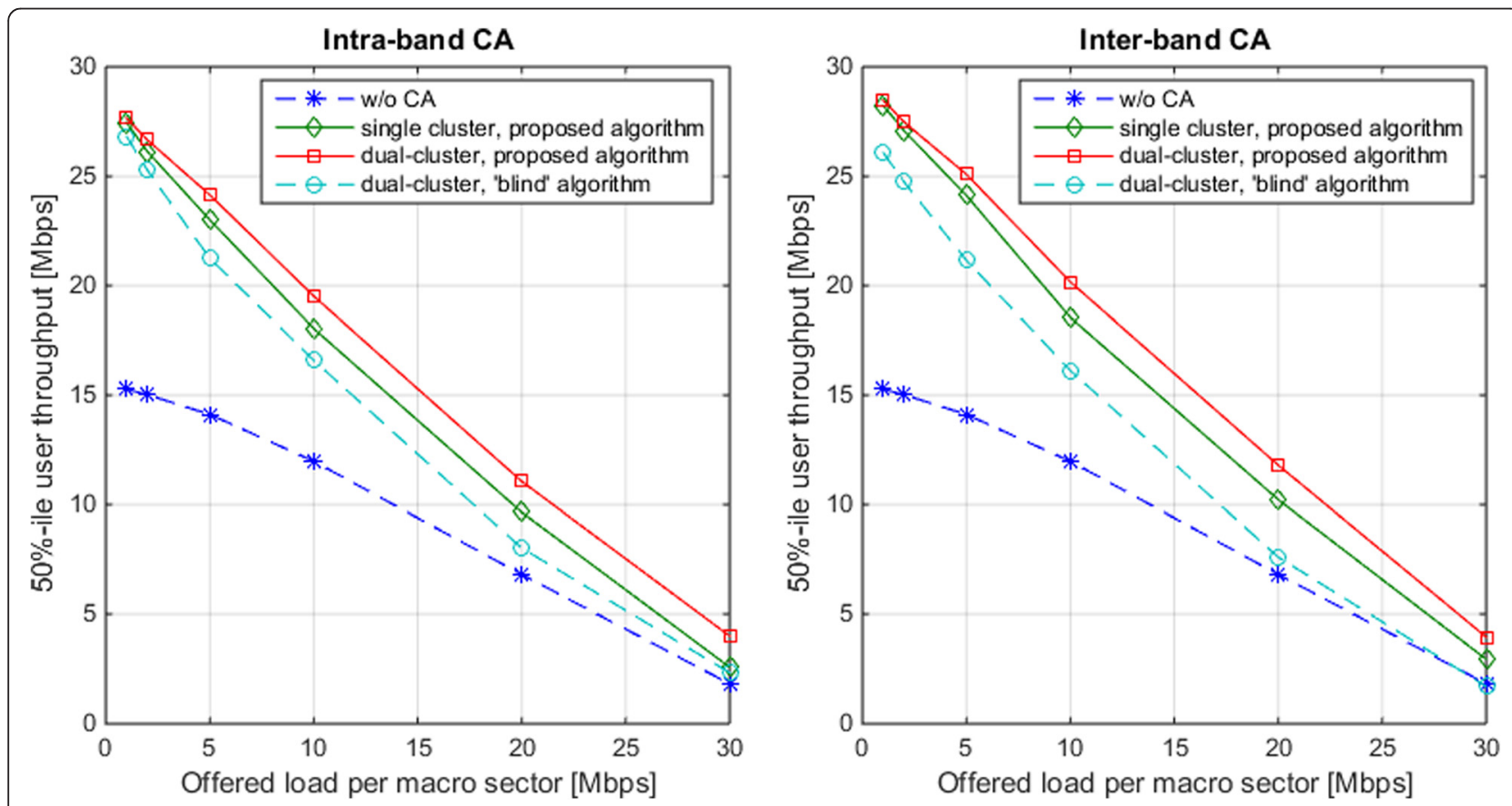

Figure 9 50th percentile user throughput under different traffic loads in different scenarios. 
and dual-cluster scheduling (i.e., increased scheduling flexibility within one CC). The power-limited cell edge users are configured only on one $\mathrm{CC}$ with single-cluster transmission so that they will not suffer from performance loss. For the case of inter-band CA, similar phenomenon can be observed.

From Figure 8 and 9, it clearly suggests avoiding using uplink CA and dual-cluster transmission at cell edge but activating them when a user is identified to be in a nonpower-limited region in order to benefit from increased transmission bandwidth and scheduling flexibility. At low load, the 50th percentile user throughput gain with $\mathrm{CA}$ as compared to the case without CA lies in the range of $70 \%$ to $80 \%$. When the load increases, the probability of a user being scheduled on both CCs is reduced, and so is the gain.

Figure 10 shows the empirical cumulative distribution function (cdf) of the average UE transmission power with 10 Mbps offered load (approximately 40\% PRB utilization) including the effect of power back-off, under different $\mathrm{CA}$ and multi-cluster transmission schemes. The curves with inter-band CA are not plotted as they are similar to the case with intra-band CA. The configuration of CA would increase the UE transmission power at the lower region of the cdf, which means that UEs not operating close to the maximum power can increase their transmission power to exploit the benefit of increased bandwidth with CA. At the higher region of the cdf, the UEs are operating in power limited conditions. With all UEs configured with CA, cell edge UEs cannot transmit with the maximum power (the maximum transmission power is $21 \mathrm{dBm}$ for cases when all UEs are configured with CA) due to the power back-off requirement specified in Equation 1. As cell edge users usually experience bad channel conditions, lowering the transmission power would decrease the SINR, thus degrading the throughput performance of those users as seen in Figure 8. With the proposed CA algorithm, the power-limited cell edge UEs are not configured with $\mathrm{CA}$ and dual-cluster transmission, thus those users do not have to back off their transmission power (the maximum transmission power with the proposed algorithm is $23 \mathrm{dBm}$ ). Therefore the transmission power with the proposed algorithm increases at the lower region, but gets closer to the case without $\mathrm{CA}$ at the higher region. Though not shown in the figure, it is observed in the study that the UE transmission power distributions without $\mathrm{CA}$ and with the proposed CC configuration algorithm do not vary much at different loads, indicating good adaptation and robustness of the LAPC algorithm and the proposed CC configuration algorithm.

\section{Conclusions}

In this paper, we have investigated the resource allocation problem for uplink CA in LTE-Advanced. For layer 3 CC configuration, we have derived a pathloss threshold that separates the UEs into two categories: power-limited and non-power-limited UEs, and assign only one CC with single-cluster transmission to power-limited LTE-A UEs but assign multiple CCs with dual-cluster transmission to non-power-limited LTE-Advanced UEs. For layer 2 packet scheduling, we have proposed an extended bandwidth expansion resource allocation algorithm with joint $\mathrm{PF}$

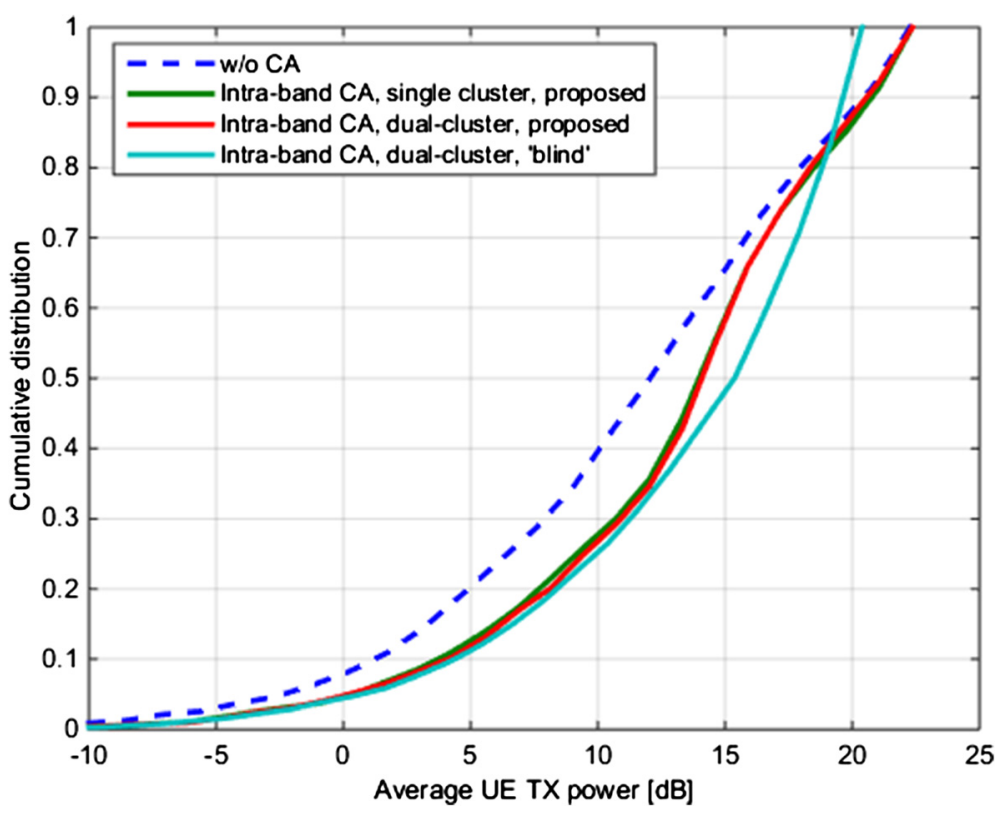

Figure 10 Average UE transmission power under different CA schemes and CC configuration algorithms, offered load =10 Mbps. 
scheduling that closely follows the envelope of the UEs' scheduling metrics. The performance of the proposed resource allocation algorithms has been evaluated for intra-band and inter-band CA via system level simulations. It is recommended that uplink CA and multi-cluster scheduling should not be configured for cell edge users, due to the limitation of UE's transmission power and the additional power back-off requirements when transmitting over non-contiguous resources. On the other hand, CA and multi-cluster scheduling should be configured for non-powerlimited UEs so that they can benefit from increased transmission bandwidth and scheduling flexibility. The results show that intra-band and inter-band CA exhibit similar trend. Specifically, with the proposed $\mathrm{CC}$ configuration algorithm, there is no loss at 5th percentile user throughput and up to $70 \%$ to $80 \%$ gain can be achieved at 50th percentile user throughput, as compared to the case without CA and dualcluster transmission. The throughput gain is especially visible at low load but decreases gradually as the load increases. Refinements of the power back-off model used for inter-band CA with dual-cluster transmission and other uplink power control methods could be interesting topics for future studies.

\section{Endnotes}

${ }^{a}$ In LTE, a PRB consists of 12 sub-carriers, each of which is $15 \mathrm{kHz}$, and is thus equal to $180 \mathrm{kHz}$.

\section{Competing interests}

The authors declare that they have no competing interests.

\section{Acknowledgement}

The authors would like to express their gratitude to Nokia Networks colleagues Huiyu Yuan, Yuyu Yan, Jens Steiner, and Mads Brix for their assistance in the development of the system-level simulator.

\footnotetext{
Author details

${ }^{1}$ Aalborg University, Fredrik Bajers Vej 7, DK-9220 Aalborg $\varnothing$, Denmark. ${ }^{2}$ Nokia Networks, Alfred Nobel Vej 21, DK-9220 Aalborg, $\varnothing$, Denmark.
}

Received: 23 September 2014 Accepted: 10 March 2015

Published online: 29 April 2015

\section{References}

1. A Ghosh, R Ratasuk, B Mondal, N Mangalvedhe, T Thomas, LTE-advanced: next-generation wireless broadband technology. IEEE Wirel. Commun. 17(3), 1536-1284 (2010)

2. G Yuan, X Zhang, W Wang, Y Yang, Carrier aggregation for LTE-advanced mobile communication systems. IEEE Wirel. Commun. 48(2), 88-93 (2010)

3. M Iwamura, K Etemad, F Mo-Han, R Nory, R Love, Carrier aggregation framework in 3GPP LTE-Advanced. IEEE Commun. Mag. 48(8), 60-7 (2010)

4. S Zukang, A Papasakellariou, J Montojo, D Gerstenberger, X Fangli, Overview of 3GPP LTE-advanced carrier aggregation for $4 \mathrm{G}$ wirelesscommunications. IEEE Commun. Mag. 50(2), 122-30 (2012)

5. K Pedersen, F Frederiksen, C Rosa, H Nguyen, L Garcia, W Yuanye, Carrier aggregation for LTE-Advanced: functionality and performance aspects. IEEE Commun. Mag. 49(6), 89-95 (2011)

6. 3GPP TS $36.213, \mathrm{v} 10.12 .0$, Release 10 physical layer procedures, March 2014 Available at www.3gpp.org/dynareport/36213.htm
7. H Holma, A Toskala, LTE for UMTS: Evolution to LTE-Advanced, 2nd edn. (John Wiley \& Sons, Chichester, UK, 2011)

8. $\quad \mathrm{L}$ Chen, $W$ Chen, $X$ Zhang, D Yang, Analysis and simulation for spectrum aggregation in LTE-Advanced system, Paper presented at the 2009 IEEE vehicular technology conference (VTC) Fall, IEEE, Anchorage, AK, 20-23 September 2009

9. Y Kakishima, T Kawamura, Y Kishiyama, H Taoka, T Nakamura, Experimental evaluation on throughput performance of asymmetric carrier aggregation in LTE-Advanced, Paper presented at the 2011 IEEE vehicular technology conference (VTC Spring),IEEE, Yokohama, 15-18 May 2011

10. H Wang, C Rosa, K Pedersen, Performance analysis of downlink inter-band carrier aggregation in LTE-Advanced, Paper presented at the 2011 IEEE vehicular technology conference (VTC Fall), IEEE, San Francisco, CA, 5-8 September 2011

11. Y Yan, A Li, A Harada, H Kayama, Enhanced downlink control channel resource allocation algorithm for cross-carrier scheduling in LTE-Advanced carrier aggregation system, Paper presented the 2011 IEEE vehicular technology conference (VTC Springer), IEEE, Budapest, 15-18 May 2011

12. H Nguyen, I Kovacs, Y Wang, K Pedersen, Feedback compression schemes for downlink carrier aggregation in LTE-Advanced, Paper presented at the 2011 IEEE vehicular technology conference (VTC Fall), IEEE, San Francisco, CA, 5-8 September 2011

13. K Yagyu, T Nakamori, H Ishii, M Iwamura, N Miki, T Asai, J Hagiwara, Investigation on mobility management for carrier aggregation in LTEAdvanced, Paper presented at the 2011 IEEE vehicular technology conference (VTC Fall), IEEE, San Francisco, CA, 5-8 September 2011

14. Y Wang, K Pedersen, P Mogensen, T Sorensen, Carrier load balancing and packet scheduling for multicarrier systems. IEEE Trans. on Wireless Communications 9(5), 1780-9 (2010)

15. W Qiu, H Minn, C Chong, Enhanced frequency diversity exploitation in carrier aggregation for LTE-Advanced systems, Paper presented at the 2011 IEEE vehicular technology conference (VTC Fall), IEEE, San Francisco, CA, 5-8 September 2011

16. A Li, K Takeda, N Miki, Y Yuan, K Hidetoshi, Search space design for cross-carrier scheduling in carrier aggregation of LTE-Advanced system, Paper presented at the 2011 IEEE international conference on communications (ICC), IEEE, Kyoto, 5-9 June 2011

17. S Shi, C Feng, C Guo, A resource scheduling algorithm based on user grouping for LTE-Advanced system with carrier aggregation. Paper presented at the 2009 international symposium on computer network and multimedia technology, IEEE, Wuhan, 18-20 January 2009

18. Y Rui, P Cheng, M Li, Q Zhang, M Guizani, Carrier aggregation for LTEadvanced: uplink multiple access and transmission enhancement features. IEEE Wirel. Commun. 20(4), 101-8 (2013)

19. F Liu, K Zheng, W Xiang, H Zhao, Design and performance analysis of an energy-efficient uplink carrier aggregation scheme. IEEE Journal on Selected Areas in Communications 32(2), 197-207 (2014)

20. R Sivaraj, A Pande, K Zeng, K Govindan, P Mohapatra, Edge-prioritized channel- and traffic-aware uplink carrier aggregation in LTE-advanced systems (IEEE International Symposium on World of Wireless, Mobile and Multimedia Networks (WoWMoM), 2012), p, 2012

21. H Wang, C Rosa, K Pedersen, Performance of uplink carrier aggregation in LTE-Advanced systems, Paper presented at the 2010 IEEE vehicular technology conference (VTC fall)IEEE, Ottawa, ON, 6-9 September 2010

22. H Wang, C Rosa, K Pedersen, Uplink component carrier selection for LTEAdvanced systems with carrier aggregation. Paper presented at the 2011 IEEE international conference on communications (ICC), IEEE, Kyoto, 5-9 June 2011

23. H Wang, C Rosa, K Pedersen, Uplink Multi-Cluster Scheduling with MU-MIMO for LTE-Advanced with Carrier Aggregation", in Proc. IEEE Wireless Communications and Networking Conference (WCNC) 2012, pp. 1-5, April 2012

24. M Lema, M Garcia-Lozano, S Ruiz, D Gonzalez, Improved component carrier selection considering MPR information for LTE-A uplink systems, in Proc. IEEE 24th International Symposium on Personal Indoor and Mobile Radio Communications (PIMRC), pp. 2191-2196, Sep. 2013

25. 3GPP TS 36.101, v11.11.0, User equipment (UE) radio transmission and reception (Release 11), Jan. 2015. Available at www.3gpp.org/dynareport/36101.htm

26. F Calabrese, C Rosa, M Anas, P Michaelsen, K Pedersen, P Mogensen, Adaptive transmission bandwidth based packet scheduling for LTE uplink, in Proc. IEEE Vehicular Technology Conference (VTC) Fall 2008, pp. 1-4, Sept. 2008 
27. C Park, L Sundström, A Wallen, A Khayrallah, Carrier aggregation for LTE-advanced: design challenges of terminals. IEEE Commun. Mag 51(12), 76-84 (2013)

28. A Nishio, T Iwai, A Matsumoto, D Imamura, System evaluation of MU-MIMO and multi-cluster allocation in LTE-Advanced uplink, in Proc. IEEE Vehicular Technology Conference (VTC) Spring 2012, pp. 1-5, May 2012

29. B Xiong, X Zhu, W Li, G Guo, Analysis of maximum power reduction of uplink for carrier aggregation in LTE-A system, in Proc. International Conference on Connected Vehicles and Expo (ICCVE) 2012, pp. 49-54, Dec. 2012

30. 3GPP TSG RAN, R4-110955, MPR for LTE multi cluster transmission, Feb. 2011

31. 3GPP TSG RAN, R4-110265, MPR for non-contiguous allocation, Jan. 2011

32. 3GPP TS 25.213, v10.0.0, E-UTRA physical layer procedures, Oct. 2010. Available at www.3gpp.org/DynaReport/25213.htm

33. C Castellanos, D Villa, C Rosa, K Pedersen, F Calabrese, P Michaelsen, J Michel, Performance of uplink fractional power control in UTRAN LTE, in Proc. IEEE Vehicular Technology Conference (VTC) Spring 2008, pp. 2517-2521, May 2008

34. M. Boussif, C Rosa, J Wigard, R Müllner, Load adaptive power control in LTE uplink, in Proc. European Wireless 2010, April 2010

35. 3GPP TSG RAN WG1 \#60bis R1-102011, Power scaling method for UL PC with carrier aggregation, April 2010

36. 3GPP TS 36.321, v10.10.0, Release 10 medium access control (MAC) protocol specification, Jan. 2014. Available at www.3gpp.org/dynareport/36321.htm

37. 3GPP TR 36.814, v9.0.0, Further advancements for E-UTRA physical layer aspects", March 2010. Available at www.3gpp.org/dynareport/36814.htm

38. ITU-R, Guidelines for evaluation of radio interface technologies for IMTAdvanced, Report ITU-R M.2135-1)", Dec. 2009. Available at www.itu.int

39. S Hämäläinen, M Reisslein, F Fitzek, A novel interface between link and system level simulations, in Proc. ACTS Mobile Telecommunications Summit, pp. 599-604, Oct. 1997

\section{Submit your manuscript to a SpringerOpen ${ }^{\circ}$ journal and benefit from:}

- Convenient online submission

- Rigorous peer review

- Immediate publication on acceptance

- Open access: articles freely available online

- High visibility within the field

- Retaining the copyright to your article 